\title{
Diseño de un Equipo para Secado Mecánico de Café y su Evaluación a Partir de la Construcción de un Modelo a Escala $1: 5$
}

\section{Designing of an Equipment for Mechanical Drying of Coffee and its Evaluation Beginning with the Construction of a 1:5 Model Scale}

José Duban Henao Cuellar $^{1}$, Luis Adolfo Perdomo Centeno ${ }^{2}$ y Luis E. Cuellar Pérez ${ }^{3}$

\begin{abstract}
Resumen
Con el fin de mejorar el proceso de secado del café, se diseñó un equipo mecánico tipo silo, el cual tiene ventajas como: menor requerimiento de espacio para secar, es desarmable, reduce la mano de obra, disminuye el tiempo de secado y conserva la calidad del producto. El silo consta de dos cámaras de secado y el flujo de aire puede circular a través de estas realizando secado ó presecado dependiendo de la dirección del flujo empleada (sistema de compuertas), con lo cual se logra homogeneidad en la humedad final del grano. Además presenta un mecanismo práctico para el descargue del grano seco, utilizando una inclinación en las camas de secado de $38^{\circ}$. El dimensionamiento del silo secador obtenido fue: Capacidad total $=2340 \mathrm{~K}$., volumen de aire $=4540.61$ $\mathrm{CFM}$, pérdidas de presión $(\Delta \mathrm{P})=2.066$ pulg. c.a., motor eléctrico monofásico de $2.5 \mathrm{HP}$. La construcción y evaluación de un prototipo a escala 1:5 del silo secador, sirvió como parámetro para obtener la siguiente información sobre la cinética del secado: humedad inicial $(50.61 \%)$, humedad final $(10.09 \%)$, temperatura promedio del aire de secado $\left(46.67{ }^{\circ} \mathrm{C}\right)$, caudal $\left(\mathrm{Q}=0.08 \mathrm{~m}^{3} / \mathrm{s}\right.$.) y tiempo de secado (28 horas). Además permitió corroborar las condiciones de operación del sistema.
\end{abstract}

Palabras clave: Silo; secado de café; tiempo de secado; contenido de humedad.

\begin{abstract}
In order to get better the process of drying the coffee, it was designed mechanic equipment like a silo which has some advantages such as: less necessity of space to dry; it is dismantled, it reduce the labor, drying duration and it keeps the product quality. The silo is constituted by two drying chambers and the airflow can go through them carrying out drying and pre-drying depending on the flow direction used ( gates system) which let homogeneity in the final humidity of the grain. Moreover, it presents a practical mechanism to download the drying grains using an inclination in the drying beds of $38^{\circ}$. The measurement of the drying silo obtained was: total capacity $=2340 \mathrm{Kg}$., air volume $=4540.61 \mathrm{CFM}$, pressure loss $(\Delta \mathrm{P})=2.066$ inches. w.g., motor single-phase electrical of $2.5 \mathrm{HP}$. The construction and evaluation of a prototype to scale 1:5 of the drying silo serves as a parameter to get the following information about the kinetics of the drying: initial moisture $(50.61 \%)$, final moisture $(10.09 \%)$, average temperature of drying airflow $\left(46.67^{\circ} \mathrm{C}\right)$, volume of flow $\left(\mathrm{Q}=0.08 \mathrm{~m}^{3} / \mathrm{s}\right.$.) and time of drying $(28$ hours $)$. Besides, it allowed to corroborate the operation conditions of the system.
\end{abstract}

Keywords: Silo; drying coffee grain; drying time; content of humidity.

1 Ph.D. en Ingeniería Agrícola. Docente Universidad Surcolombiana Neiva. Avenida Pastrana Carrera 1'.duvanhenao23@ hotmail.com

2 Ingeniero Agrícola. Universidad Surcolombiana Neiva. Avenida Pastrana Carrera 1. luad4pece@hotmail.com

3 Ingeniero Agrícola. Universidad Surcolombiana Neiva. Avenida Pastrana Carrera 1 ${ }^{a}$.enriquecuellarl@ @otmail.com 


\section{Introducción}

En la mayoría de las zonas cafeteras, el secado se realiza de forma natural utilizando energía solar como fuente de calor, lo que genera varios inconvenientes que dificultan dicha tarea. Entre estos se encuentran la falta de infraestructura adecuada, condiciones climáticas adversas que habitualmente coinciden con los periodos críticos de lluvia y pocas horas de brillo solar durante la cosecha, impidiendo que los patios de secado tengan buenos rendimientos y permitan el secado eficaz y eficiente. De esta manera suelen acumularse grandes cantidades de café que no pueden ser ubicadas en los patios de secado. Cuando el café pergamino después de cosechado se deja por tiempos prolongados con su contenido inicial de humedad 52 al $56 \%$ bh, por retraso en el proceso de secado o cuando este se almacena con contenidos de humedad superiores al $12 \%$ bh debido a un proceso de secado deficiente, el riesgo de ser atacado por hongos y ser contaminado con micotoxinas es muy alto. La contaminación del grano hace que éste pierda su calidad, ocasionando su rechazo en los mercados nacionales e internacionales, lo cual afecta drásticamente los ingresos de los caficultores y del país (Coronado et al., 2008).

El proceso de secado se da en el momento que se presenta transferencia de calor y masa, como son la humedad interna ó vapor dentro del sólido y líquido evaporado de las superficies húmedas del mismo. Unos de los mecanismos que controlan el libre movimiento de la humedad en la parte interna son su difusión como líquido ó vapor, la acción capilar, el encogimiento y los gradientes de presión, gravedad y vaporización de la humedad (Solano, 2002).

El secado en capas profundas es uno de los sistemas más usados para secado de productos biológicos dadas las características y necesidades del mismo, a mayor profundidad, mayor sobresecamiento para un flujo de aire específico. Para espesores de capa de grano iguales o inferiores a 0.45 metros, se recomienda temperatura del aire de secado superior a $43^{\circ} \mathrm{C}$ (Brooker et al, 1992).

Con el avance tecnológico cada día aumentan proporcionalmente los sistemas de secado mecánico, por razones de disminución de riesgos, costos y mejor control de la producción. Si se desea que los sistemas de secado de café, o de cualquier grano, sean eficientes, se debe conocer la manera de operarlos adecuadamente para preservar las características físicas, químicas y sensoriales de los productos agrícolas, especialmente las del café, se ha encontrado que la manera más efectiva de lograrlo es mediante el secado (Roa et al., 1999). Con la implementación de un equipo para el secado mecánico de café se presenta una alternativa de secado a pequeños y medianos productores, con los cuales se puede obtener ventajas como menor requerimiento de espacio para secar, el equipo es desarmable por lo cual puede ser transportado de un lugar a otro con facilidad, reducción de la mano de obra, se puede obtener mayor volumen de grano seco, no depende de las condiciones climáticas, es operable las 24 horas del día, disminución del tiempo de secado y conservación de la calidad, de tal manera que permita obtener con menos riesgo café tipo exportación que cumpla con los estándares de calidad exigidos por los mercados internacionales.

En el presente artículo se muestra de manera clara y detallada el diseño de un silo secador de café y la evaluación a partir de la construcción de un modelo a escala 1:5. Este consta de dos cámaras de secado, sobre las cuales el flujo de aire puede circular en dos sentidos haciendo secado y presecado sobre la capa de grano, con el objetivo de garantizar un secado más homogéneo. Este sistema de silo mecánico cuenta con un medio sencillo de compuertas para permitir la inversión de sentido del flujo de aire y de igual manera presenta un mecanismo práctico para el descargue del grano seco, disminuyendo costos en personal para realizar esta labor.

\section{Metodología}

\subsection{Parámetros de diseño}

El sistema está compuesto por una unidad de secado (silo), un ventilador centrífugo con alabes inclinados hacia atrás y requiere de un intercambiador de calor.

El equipo fue dimensionado para satisfacer los requerimientos de secado de $2340 \mathrm{Kg}$. de café por turno de secado utilizando los intervalos de tiempo más adecuados para realizar la inversión del sentido del flujo de aire en este tipo de secador, donde se tienen $1170 \mathrm{Kg}$. de grano en secado y 1170 en presecado dependiendo de la dirección de flujo que se opere en el momento, de tal manera que se logre obtener un producto con buena uniformidad en el contenido de humedad final (dentro del rango 10 a $12 \%$ bh). Este dimensionamiento se hizo con el objetivo de hacer un uso adecuado de los materiales de construcción y de acuerdo a la producción anual de café en las fincas. Según ROA et al. (1999), la producción de pequeños caficultores es de hasta 6.25 ton cps/año y medianos es de 6.25 hasta

12.5 ton cps/año. 
En cuanto al tiempo de secado, empleando la temperatura recomendada de diseño $\left(50{ }^{\circ} \mathrm{C}\right)$ se puede retirar el contenido de humedad inicial del café (50 a 55\% bh) hasta llevarlo a un rango del 10 a $12 \%$ bh, en un tiempo estimado de 24 a 28 horas, garantizando la calidad del producto. Para temperaturas de secado menores este tiempo puede incrementarse significativamente.

Para la operación de este equipo se debe cargar cada una de sus camas con la totalidad del volumen de café para el cual fueron diseñadas y durante el periodo de secado se debe realizar inversión del flujo de aire caliente cada 4 ó 6 horas, para garantizar el secado homogéneo del grano. A continuación se presenta mediante las Figuras 1,2 y 3, el diseño del silo secador:

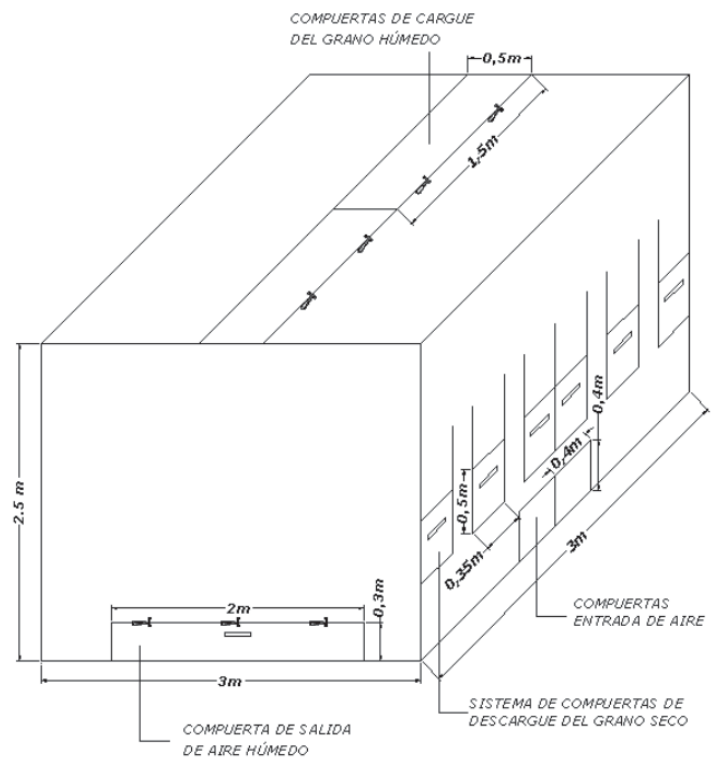

Figura. 1. Vista ortogonal externa del silo secador

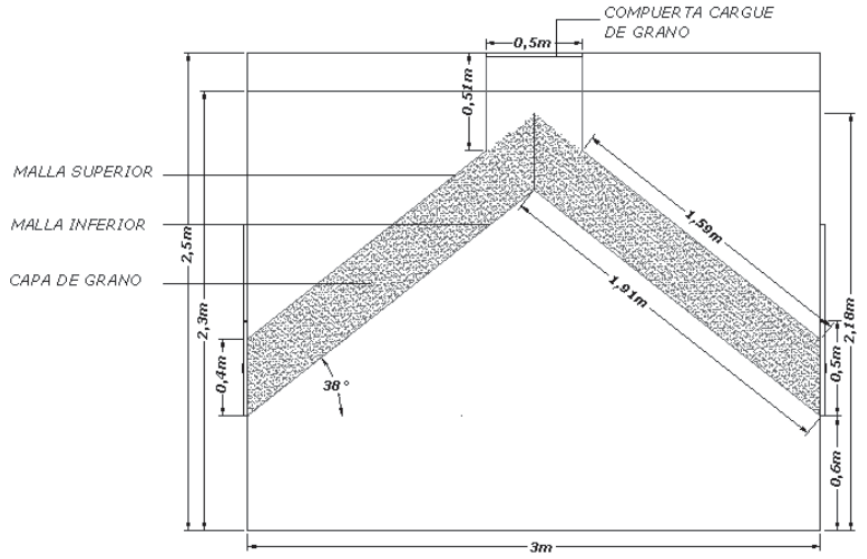

Figura. 2. Vista corte lateral del silo secador

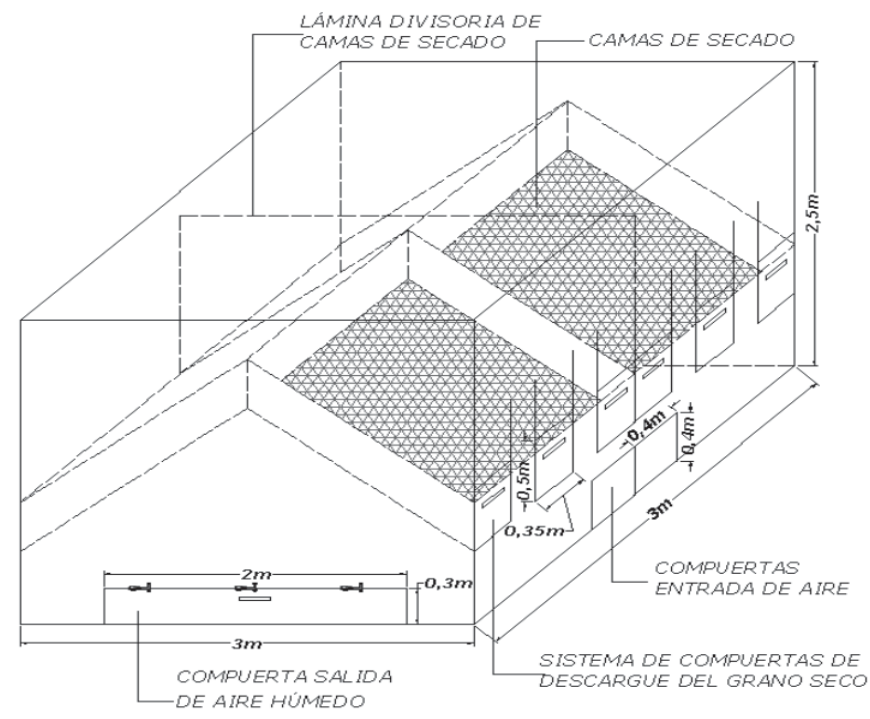

Figura 3. Vista ortogonal interna del silo secador 


\subsection{Descripción del equipo de secado y su funcionamiento}

Los materiales de construcción del silo secador son totalmente metálicos. Este consta de tres componentes fundamentales: ventilador centrífugo, intercambiador de calor y el cuerpo de secado, que consta de dos camas para grano (cada una con dos módulos), dos compuertas de distribución de aire, dos para la salida del aire húmedo y 12 para descargar el grano.

Para evitar daños en el grano, la temperatura no debe exceder los $50{ }^{\circ} \mathrm{C}$. De igual manera el caudal del flujo de aire debe ser adecuado para el volumen de café a secar. De acuerdo a ROA M., et al. (2000), para capas estáticas el caudal recomendado es de $25 \mathrm{~m}^{3}$ por minuto por cada metro cúbico ocupado por el grano.

La Figura 4 representa el principio de funcionamiento del silo secador:

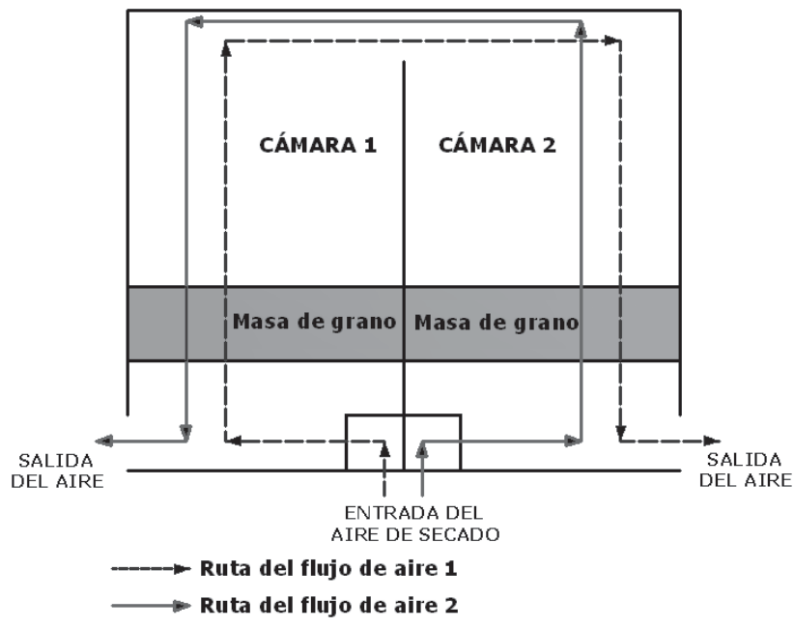

Figura 4. Esquema recorrido del flujo de aire a través del silo secador

\subsection{Dimensionamiento de componentes del silo}

Los principales componentes del silo secador son el cuerpo de secado, unidad de ventilación y unidad calorífica.

\subsubsection{Cuerpo de secado}

- Dimensiones del silo secador. El silo es de forma cuadrada, con dimensiones 3 x 3 en su base y altura total de $2.5 \mathrm{~m}$. En la Tabla 1 se expresan todas las distancias desde la base hasta la parte superior del silo, donde se tuvo en cuenta el espesor que ocupa la capa de grano y el grado de inclinación de la misma $\left(38^{\circ}\right)$.

Tabla 1. Dimensiones del silo secador

\begin{tabular}{|l|c|}
\hline \multicolumn{1}{|c|}{ Detalle } & Dimensión $(\mathbf{m})$ \\
\hline Largo & 3.0 \\
\hline Ancho & 3.0 \\
\hline Altura de la malla inferior & 0,6 \\
\hline Altura total de la cama inclinada & 2.18 \\
\hline Altura de la compuerta superior de paso de aire & 2.3 \\
\hline Altura total del silo & 2,5 \\
\hline
\end{tabular}

- Materiales de construcción del silo secador. De acuerdo con MOTTA Y REPIZO (1982), se recomienda el método de H.A. Janssen. Mediante esta metodología, se determinó el espesor de lámina para el diseño y el número 
De tornillos con su espaciamiento para unir las láminas entre sí. Para obtener los perfiles del marco estructural que sostienen el peso del grano, de la estructura y cargas vivas, así como los perfiles de soporte estructural y amarre perimetral del silo secador, se empleó la metodología de GARCIMARTíN (1998). (Ver Tabla 2). Los marcos estructurales de la malla inferior y superior se realizaron en forma de cuadrícula 50 x $50 \mathrm{~cm}$., utilizando el perfil seleccionado (ver Figura 5).

Tabla 2. Materiales seleccionados mediante el análisis estructural

\begin{tabular}{|l|c|}
\hline \multicolumn{1}{|c|}{ Materiales } & Dimensión \\
\hline Lámina calibre $20($ espesor $0.9 \mathrm{~mm})$ & $3 \times 1 \mathrm{~m}$ \\
\hline Lámina troquelada calibre $20(\varnothing=4 \mathrm{~mm})$ & $3 \times 1 \mathrm{~m}$ \\
\hline Perno de alta resistencia $($ Espaciamiento $=0.33 \mathrm{~m})$ & $1 / 4 \times 1 ”$ \\
\hline Perfil marco estructural de soporte malla inferior & $40 \times 40 \times 4 \mathrm{~mm}$ \\
\hline Perfil marco estructural de soporte malla superior & $19 \times 19 \times 2.5 \mathrm{~mm}$ \\
\hline Ángulo de amarre perimetral y soporte estructural & $40 \times 40 \times 4 \mathrm{~mm}$ \\
\hline
\end{tabular}

2.3.2 Selección del ventilador. Conociendo el caudal de aire, pérdidas de presión y la potencia del motor, fue seleccionado el ventilador.

- Cálculo del flujo o caudal de aire del ventilador. El cálculo se realizó teniendo en cuenta la velocidad del aire de secado dentro del silo recomendada por Roa et al. (1988). También se calculó teniendo en cuenta el caudal recomendado por CENICAFE y mediante el número de Bushels (Brooker et al, 1992). En la Tabla 3 están los resultados de caudal calculado mediante cada criterio.

Tabla 3. Criterios aplicados para estimar el caudal para el silo secador

\begin{tabular}{|l|c|}
\hline \multicolumn{1}{|c|}{ Criterio } & Caudal (CFM) \\
\hline Velocidad del aire de secado $(28.5 \mathrm{~m} / \mathrm{min})$ & 4529.1 \\
\hline Caudal recomendado por Cenicafé $(25 \mathrm{~m} 3 / \mathrm{min} / \mathrm{m} 3$ de grano) & 4540.46 \\
\hline Número de Búshels & 4540.61 \\
\hline
\end{tabular}

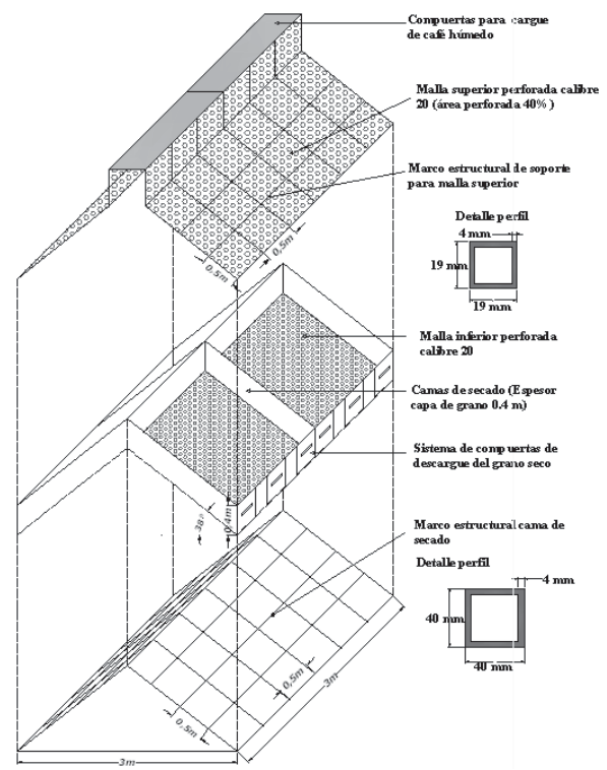

Figura 5. Disposición de las mallas y marco estructural de las camas de secado 
- Determinación de pérdidas de presión en el sistema. Las pérdidas se calcularon teniendo en cuenta que estas se presentan principalmente en las cámaras, capas de grano, ductos, compuertas, lámina perforada y expansiones. Para el diseño se obtuvo una pérdida de presión total de 2.066 Pulg. c.a. Ver Tabla 4.

Tabla 4. Pérdidas totales en el sistema

\begin{tabular}{|c|c|c|c|c|c|}
\hline $\begin{array}{c}\Delta \text { P Grano(Pulg. } \\
\text { ca) }\end{array}$ & $\begin{array}{c}\Delta \mathbf{P} \text { Malla (Pulg. } \\
\text { ca) }\end{array}$ & $\begin{array}{c}\Delta \text { P Ducto(Pulg. } \\
\text { ca) }\end{array}$ & $\begin{array}{c}\Delta \mathbf{P} \text { Exp. } \\
\text { Abrupta (Pulg. } \\
\text { ca) }\end{array}$ & $\begin{array}{c}\Delta \text { P Orif (Pulg. } \\
\text { ca) }\end{array}$ & $\Delta$ Pt Estática \\
\hline 1.44 & 0,038 & 0,0184 & 0,35 & 0.22 & 2.066 \\
\hline
\end{tabular}

- Potencia del motor. Se determinó luego de conocidos los valores de caudal $(\mathrm{Q})$, pérdida de presión total $(\Delta \mathrm{Pt})$ y la eficiencia del ventilador, obteniendo una potencia de $2.5 \mathrm{HP}$ para el motor.

\subsection{Construcción del prototipo a escala 1:5}

El prototipo se construyó con base en los materiales y características del diseño original del silo secador, lámina cold roll calibre 20, lámina perforada cold roll calibre 20 con perforaciones de $4 \mathrm{~mm}$ de diámetro. Para simular el intercambiador de calor y el ventilador se utilizó una resistencia de 1000 Watt y dos ventiladores en serie de flujo axial de $\Phi=4 \frac{1}{2} "$.

\subsection{Evaluación del prototipo}

Para efectuar la evaluación y encontrar las condiciones de funcionamiento del silo secador, se tuvieron en cuenta las características del producto a secar, del lugar donde se efectuaron las pruebas y las características del prototipo a escala 1:5 (caudal y temperatura del aire de secado). El proceso de secado en el prototipo se hizo, realizando inversión del sentido del flujo de aire cada 4 horas y se tomaron datos cada 2 horas.

\section{Resultados}

3.1 Determinación de humedad inicial del café. La determinación del contenido inicial de humedad se hizo de acuerdo con la norma ISO 6673, para determinación de humedad inicial de productos biológicos (ver Tabla) 5.

Tabla 5. Determinación de Hbh y Hbs del café

\begin{tabular}{|c|c|c|c|c|c|c|}
\hline Muestra & Winicial (g) & Wfinal (g) & $\begin{array}{c}\text { \%Humedad } \\
\text { B. H. }\end{array}$ & $\begin{array}{c}\text { Prom. \% } \\
\text { Humedad B. } \\
\text { H. }\end{array}$ & $\begin{array}{c}\text { \% Humedad } \\
\text { B. S. }\end{array}$ & $\begin{array}{c}\text { Prom. \% } \\
\text { Humedad } \\
\text { B.S. }\end{array}$ \\
\hline 1 & 116.48 & 57.78 & 50.39 & & 101.59 & \\
\hline 2 & 110.40 & 54.73 & 50.43 & & 101.72 & \\
\hline 3 & 113.15 & 55.90 & 50.60 & 50.61 & 102.41 & 102.49 \\
\hline 4 & 114.73 & 56.61 & 50.66 & & 102.66 & \\
\hline 5 & 110.87 & 54.33 & 51.00 & & 104.08 & \\
\hline
\end{tabular}

3.2 Proceso de secado en el prototipo silo secador. En el momento de iniciar el proceso de secado la temperatura ambiente fue de $27^{\circ} \mathrm{C}$, humedad relativa del $67 \%$ y la temperatura de entrada al silo $45^{\circ} \mathrm{C}$. El flujo de aire se dirigió hacia una de las cámaras por medio de la apertura o cierre de compuertas, donde la primera capa de grano $(8 \mathrm{~cm})$ que atravesó actuó en secado y la otra en presecado. Al invertir el sentido del flujo de aire en el otro ciclo la primera capa que inicialmente fue de secado luego estuvo en presecado. Este proceso permitió que no se presentaran diferencias significativas en el gradiente de humedad final entre el grano ubicado en el punto inferior de la cama hasta el que estuvo ubicado en la parte superior. 


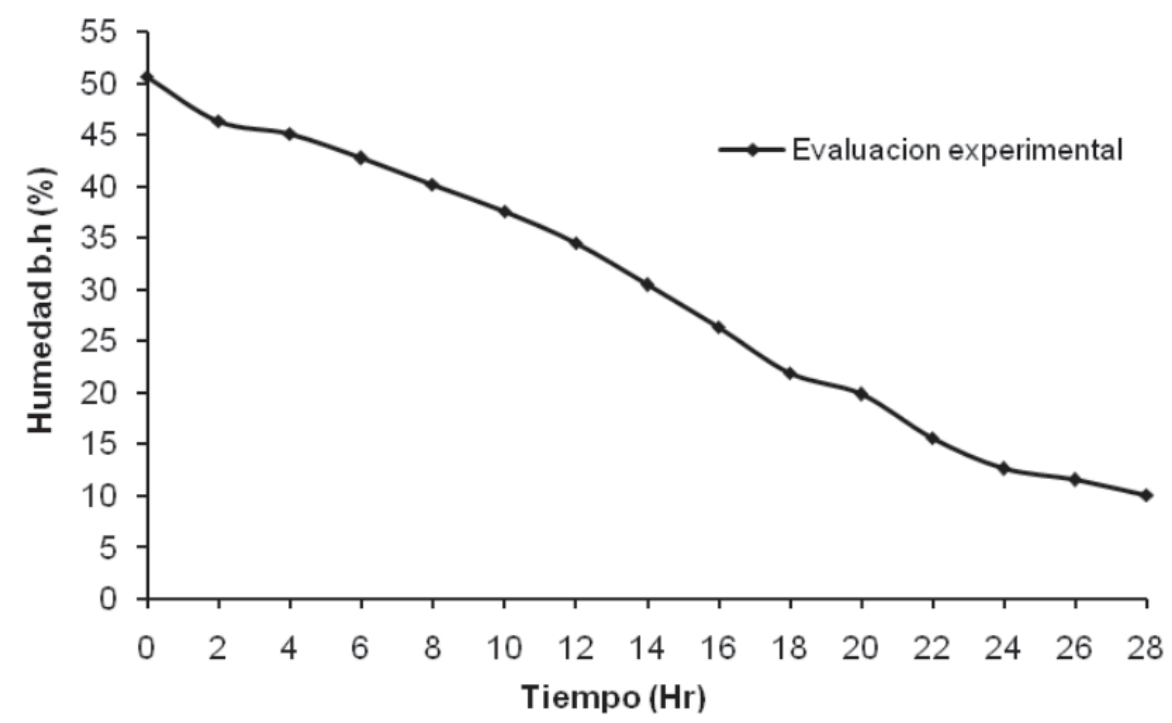

Figura 6. Comportamiento de la humedad durante el proceso de secado

Con las pruebas efectuadas en el prototipo a escala 1:5, se determinó que durante todo el proceso de secado el porcentaje de humedad removida del grano en el interior del silo se realizó lentamente, debido a que las temperaturas fueron bajas y variables (promedio de $46.67^{\circ} \mathrm{C}$ ) y no permitieron el retiro constante de humedad como muestra la Figura 6, así como ocurre a condiciones ideales (modelación matemática de Roa) ver Figura 7.

El tiempo de secado obtenido en el ensayo fue satisfactorio, ya que se aproxima a la curva de modelación de secado de Roa, la cual predice el tiempo para las condiciones de trabajo (temperatura de $50{ }^{\circ} \mathrm{C}$ y una capa de grano de $0,40 \mathrm{~m})$.

Al igual comparando este resultado con estudios de evaluación y modelación de CORONADO et al (2008), los cuales realizaron ensayos comparando el secado experimental de silos, conformados por cámara de secado y presecado, con modelaciones matemáticas encontraron que el tiempo de secado para llevar la humedad del 50 al $12 \%$ es de 23 horas en secado y 32 horas en presecado; de igual manera ROA, et al. (2000), en el avance técnico 282 de CENICAFÉ, establece que para una temperatura de secado de $50^{\circ} \mathrm{C}$ y un espesor de capa de café entre 0,1 y $0,4 \mathrm{~m}$ se obtienen tiempos de secado total entre 20 y 24 horas con el contenido de humedad final entre el 11 y $16 \%$.

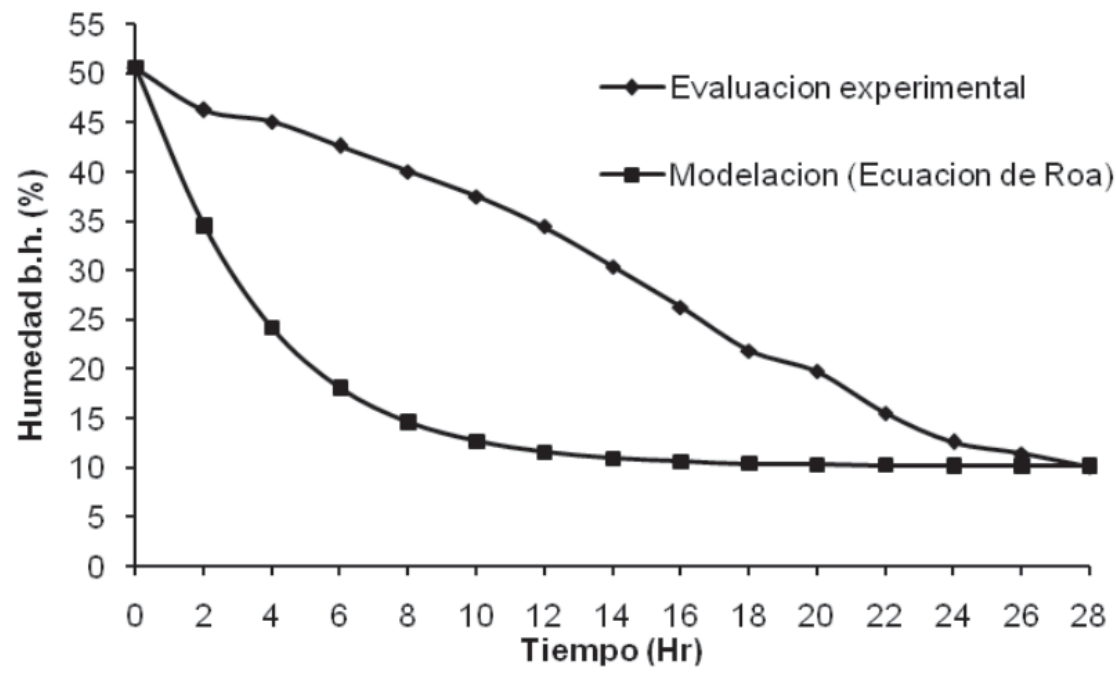

Figura 7. Comparación curva de secado experimental Vs. Modelación 


\section{Conclusiones y recomendaciones}

Las características finales de diseño del silo secador de dos cámaras de secado con inversión de flujo, son: capacidad volumétrica total (3.6 m3), capacidad másica (\$2.34 Ton de café húmedo), temperatura del aire de secado $\left(50{ }^{\circ} \mathrm{C}\right)$, espesor de capa de grano $(\quad 40 \mathrm{~cm})$, caudal de aire $\quad(4540.61 \mathrm{CFM})$, presión estática ( $\quad 2.066$ Pulg.ca) y potencia del motor $(2.5 \mathrm{HP})$.

La malla (área perforada del 40\%) ubicada en la parte superior del silo, controla la uniformidad en el espesor de capa y también facilita el cargue y acomodamiento del grano dentro del silo.

El equipo permite realizar su transporte con facilidad, ya que se encuentra diseñado en lámina cold roll calibre 20 unidas entre sí utilizando pernos de 1 x 1/4" y ángulos de amarre perimetral de 40 x 40 x $4 \mathrm{~mm}$, lo que hace que sea desarmable.

El prototipo del silo secador construido a escala 1:5, permitió evaluar la cinética del secado del grano con los siguientes resultados: Humedad inicial $(50.61 \%)$, humedad final $(10.09 \%)$, temperatura promedio del aire de secado $\left(46.67^{\circ} \mathrm{C}\right)$, caudal $\left(\mathrm{Q}=0.08 \mathrm{~m}^{3} / \mathrm{s}\right.$. $)$ y tiempo de secado $(28$ horas $)$.

A partir de la evaluación del prototipo silo secador, se comprobó la efectividad del sistema de descargue del grano empleando una inclinación de $38^{\circ}$, representando una ventaja operativa que reduce los costos al caficultor.

\section{Referencias Bibliográficas}

1. Brooker D.B, Bakker-Arkema, F.W.; Hall, C.W., 1992. Drying and storage of grains and oilseeds. Westport: The Avi publishing company, 450p.

2. Coronado P., Alfonso; ROA M., Gonzalo y Tascón O., Carlos.,2008. Recomendaciones para el manejo eficiente de los secadores mecánicos de café pergamino, (Consulta: 02 de Julio de 2009), <www.agriambi.com.br〉.

3. Garcimartin, Miguel Ángel., 1998. Edificación agroindustrial: Estructuras metálicas, ediciones MundiPrensa, Madrid-España, 90p.

4. Motta, Luis y Repizo, Orlando.,1982. Diseño y construcción de un secador para granos. Trabajo de grado Ingeniería Agrícola. Neiva: Universidad Surcolombiana. Facultad de ingeniería,132p.

5. Roa M., Gonzalo, et al. Beneficio ecológico del café.,1999. Primera edición. CENICAFE, Chinchiná, 300p.

6. Roa M., Gonzalo, et al., 2000.Secado mecánico del café, CENICAFE, avance técnico 282, Chinchiná, 8p.

7. Roa M., Gonzalo, et al., 1988.Tecnología del cultivo del Café: Secado mecánico del café, CENICAFE, Manizales- caldas, Segunda Edición. p 311-340. 404p.

8. Solano Osorio, Jair Fernando.,2002. Diseño, construcción y evaluación de un secador desarmable con destino a pequeños y medianos caficultores. Trabajo de grado Ingeniero Agrícola. Neiva: Universidad Surcolombiana. Facultad de ingeniería, 81p. 